Int J Infect Microbiol 2012;1(1):1-2

(C)2012 GLR All Rights Reserved, eISSN: 2091-2145

DOI: http://dx.doi.org/10.3126/ijim.v1i1.6937

\section{Rapid HIV diagnostics}

\section{Afridi NK}

Health Department, Government of Khyber, Pakhtunkhwa, Pakistan

email: naseemkafridi@hotmail.com, Tel. No.: (+92)3018933573
HIV/AIDS is still a formidable challenge for human race both in terms of development and mortality. Researchers and scientists have been trying hard to find diagnostic tools for its early detection so as to reduce morbidity and mortality from it. So far they have been successful but are still striving to find more accurate and rapid HIV detection test and considering cost effectiveness of the tests. It is of much public health significance to detect HIV infection in acute stage because this stage is highly infectiousness and appears to account for a disproportionate amount of HIV transmission. The latest advances in HIV testing, including thirdgeneration enzyme immunoassays (EIAs) that detect anti-HIV IgM and IgG antibodies, fourthgeneration combination EIAs that detect both antiHIV antibodies and HIV p24 antigen, and nucleic acid-based testing for HIV RNA, have obviously decreased the time interval between infection and detection of infection. The availability and wide use of these latest technologies have motivated review of recommended HIV testing algorithms. A persons' knowledge of their HIV sero-status contributes to reducing transmission risk behaviors. Thus, widespread testing, facilitated by the latest technology, permits more people to identify their sero-status and is the first step in any successful effort to control the incidence of HIV infection.

Available rapid diagnostic tests using samples of oral fluid, whole blood, plasma, or serum are lateral flow devices able to detect anti-HIV IgG and IgM but not HIV antigen. The sensitivities and specificities of available lateral flow devices range from $99.3 \%$ to $100 \%$ and $99.7 \%$ to $99.9 \%$, respectively, with narrow 95\% confidence intervals, per the product information of these rapid diagnostics. Newer diagnostic techniques permit earlier detection of HIV infection during acute infection than does Western blot testing. After infection, symptoms may appear within 2 weeks. HIV p24 levels (measurable with fourthgeneration EIAs) typically peak after the onset of symptoms, at about 2.5 weeks to 3 weeks after infection; plasma HIV RNA levels (measurable by nucleic acid amplification tests (NAATs) begin to increase at about 1.5 weeks to 2 weeks, peaking at around 3 weeks to 6 weeks after infection. With the subsequent occurrence of antibody response, third-generation EIAs can detect antibody as early as 3 weeks to 4 weeks after infection, and secondgeneration tests can return positive results at 
around 4.5 weeks to 5 weeks after infection. By comparison, Western blot testing first begins to show positive results at around 5 weeks. Direct comparison of some of the newer techniques with Western blot testing has shown that positive results are obtained days to weeks before the Western blot test yields positive or even indeterminate results. Indeterminate Western blot test results are frequently associated with detection of anti-p24 antibody in the setting of both false-positive and negative screening EIAs. Indeed, anti-p24 antibody is the most commonly detected antibody in the setting of false positive EIAs and indeterminateresult Western blots. ${ }^{1}$ The fact that rapid HIV testing techniques do not include a p24 assay may allow such tests to avoid a proportion of false-positive results.

The potential value of rapid testing in this respect is illustrated by findings in a study in which women in labor for whom no HIV test results were available were screened. ${ }^{2}$ Among 7680 women screened, 54 $(0.7 \%)$ new HIV infections were identified. Oral rapid testing yielded 6 false-positive results and no false-negative results. EIA testing yielded 15 falsepositive results, of which 7 were positive for p24 only and 8 were negative on Western blot testing. Overall, specificities for the oral rapid test and the EIA were $99.9 \%$ and $99.0 \%$, respectively, and positive predictive values were $90 \%$ and $76 \%$, respectively. Such findings indicate that p24excluding rapid tests may be less prone to falsepositive results in the labor and delivery setting, potentially avoiding unnecessary exposure of mothers and infants to antiretroviral drugs.

\section{REFERENCES}

1. Midthun K, Garrison L, Clements ML, Farzadegan H, Fernie B, Quinn T. Frequency of indeterminate Western blot tests in healthy adults at low risk for human immunodeficiency virus infection. The NIAID AIDS Vaccine Clinical Trials Network. J Infect Dis 1990;162:1379-1382.

2. Bulterys M, Jamieson DJ, O'Sullivan MJ, et al. Rapid HIV-1 testing during labor: a multicenter study. J Am Med Assoc 2004;292:219-223. 